\title{
Isotope effects in the photofragmentation of symmetric molecules: The branching ratio
} of $\mathrm{OD} / \mathrm{OH}$ in water

\author{
Henriksen, Niels Engholm; Møller, Klaus Braagaard; Engel, Volker
}

Published in:

Journal of Chemical Physics

Link to article, DOI:

$10.1063 / 1.1924409$

Publication date:

2005

Document Version

Publisher's PDF, also known as Version of record

Link back to DTU Orbit

Citation (APA):

Henriksen, N. E., Møller, K. B., \& Engel, V. (2005). Isotope effects in the photofragmentation of symmetric molecules: The branching ratio of OD/OH in water. Journal of Chemical Physics, 122(20), 204320.

https://doi.org/10.1063/1.1924409

\section{General rights}

Copyright and moral rights for the publications made accessible in the public portal are retained by the authors and/or other copyright owners and it is a condition of accessing publications that users recognise and abide by the legal requirements associated with these rights.

- Users may download and print one copy of any publication from the public portal for the purpose of private study or research.

- You may not further distribute the material or use it for any profit-making activity or commercial gain

- You may freely distribute the URL identifying the publication in the public portal 


\title{
Isotope effects in the photofragmentation of symmetric molecules: The branching ratio of $\mathrm{OD} / \mathrm{OH}$ in water
}

\author{
Niels E. Henriksen and Klaus B. Møller ${ }^{a)}$ \\ Department of Chemistry, Technical University of Denmark, DK-2800 Kgs. Lyngby, Denmark \\ Volker Engel \\ Institut für Physikalische Chemie, Universität Würzburg, Am Hubland, D-97074 Würzburg, Germany
}

(Received 1 February 2005; accepted 30 March 2005; published online 25 May 2005)

\begin{abstract}
With HOD initially in its vibrational ground state, we present a new detailed interpretation of the $\mathrm{OD} / \mathrm{OH}$ branching ratio $(\sim 3)$ in the photoinduced process $\mathrm{D}+\mathrm{OH} \leftarrow \mathrm{HOD} \rightarrow \mathrm{H}+\mathrm{OD}$, in the first absorption band. Using semiclassical arguments, we show that the branching ratio has little to do with the initial distribution of configurations, but the initial momentum distribution plays a key role in determination of the branching ratio. The formation of $\mathrm{D}+\mathrm{OH}$ arises from initial situations where $\mathrm{OD}$ is stretching, and it stretches faster than $\mathrm{OH}$, whereas all other motions lead to $\mathrm{H}+\mathrm{OD}$. This picture is confirmed by quantum wave-packet calculations. (C) 2005 American Institute of Physics. [DOI: 10.1063/1.1924409]
\end{abstract}

\section{INTRODUCTION}

In a chemical reaction, the branching ratio between chemically distinct products is clearly a very important fundamental quantity. Since laser light can be used to steer chemical reactions, ${ }^{1-3}$ i.e., guide a system into a desired final state, many theoretical studies have now been devoted to the control of branching ratios in photoinduced reactions. In addition, over the last decade it has become experimentally feasible to generate laser fields with complex pulse shapes. With the possible exception of the high intensity limit, the outcome of the interaction between matter and light can conveniently be described as an interplay between the field-free molecular dynamics and the field. Thus, it is important to understand the molecular dynamics in situations where chemically distinct products can show up.

A simple example of a photoinduced reaction with chemically distinct products is the two-body dissociation of HOD in the first absorption band: ${ }^{4}$

$$
\mathrm{HOD} \rightarrow\left\{\begin{array}{l}
\mathrm{H}+\mathrm{OD} \\
\mathrm{D}+\mathrm{OH}
\end{array} .\right.
$$

The control of the branching ratio between the two channels in this reaction has attracted much interest both experimentally and theoretically. ${ }^{5-12}$ Spurred by this interest we will in this paper analyze the origin of the "natural" branching ratio (to be defined below) for this reaction. In the following, $P_{\mathrm{H}}$ $\left(P_{\mathrm{D}}\right)$ denote the probability for the upper (lower) reaction in Eq. (1).

With HOD in its vibrational ground state, it has been found in theoretical ${ }^{13}$ as well as experimental studies ${ }^{14}$ that bond breaking of $\mathrm{H}-\mathrm{OD}$ is preferred over the breaking of HO-D. These studies consider excitation by ultraviolet $\mathrm{cw}$ (continuous wave) lasers-or laser pulses with a long dura-

\footnotetext{
${ }^{a)}$ Author to whom correspondence should be addressed. FAX: $(+45) 4588$ 3136. Electronic mail: klaus.moller@kemi.dtu.dk
}

tion compared to the relevant nuclear dynamics, and at all wavelengths within the first absorption band (with a maximum at $\lambda \sim 166 \mathrm{~nm}$ ) it was found that the branching ratio, $P_{\mathrm{H}} / P_{\mathrm{D}}$, is larger than 2 and less than about $4-5$, except at the wings of the absorption band where the dissociation probability is extremely low. The question we are considering is how to understand/interpret this result. This question has been addressed previously, ${ }^{5,13,15}$ but, as we will demonstrate in this paper, none of these works presented a completely coherent explanation.

We consider in the following not the excitation by $\mathrm{cw}$ lasers (or long pulses) but rather the branching ratio obtained after excitation by a pulse that is infinitely short in time (a $\delta$ pulse). In this (theoretical) limit the laser pulse simply promotes to the excited electronic state a replica of the initial nuclear state-times the transition dipole moment of the electronic transition-which then evolves under field-free conditions (a Franck-Condon wave packet). ${ }^{16,17}$ This provides a simple picture of the photoinduced dynamics and it can be shown that the branching ratio dictated by the Franck-Condon wave packet is identical to the branching ratio obtained by a cw laser, when the full absorption band is scanned uniformly over all frequencies. ${ }^{9}$ In fact, the dynamics created by any laser-pulse shape - in the weak-field (firstorder perturbation theory) limit — can be described as a superposition of Franck-Condon wave packets excited at different times. It can be shown that the pulse shape has a rather "trivial" effect on the branching ratio. ${ }^{17,18}$ Thus, in the final product distributions obtained with shaped laser pulses, a number of energy states are simultaneously excited, but in such a way, that the same distribution could have been obtained via a set of consecutive processes induced by cw lasers with the same frequency distribution as in the shaped pulse. The coherence established among the Franck-Condon wave packets plays no role for the branching ratio. It is in this sense, we denote the branching ratio dictated by the Franck-Condon wave packet the natural branching ratio. 
Previous attempts to rationalize that $\mathrm{H}+\mathrm{OD}$ is the preferred channel were also, to some extent, based on the dynamics of the Franck-Condon wave packet. ${ }^{5,13,15}$

As in the paper by Engel and Schinke ${ }^{13}$ we analyze the dynamics of the Franck-Condon wave packet using the (semi-) classical Wigner method. ${ }^{15,17,19-21}$ The Wigner method allows us to describe the time evolution of a system in terms of a swarm of trajectories following an exact sampling of the initial quantum state. Of course, such a description is not always accurate, but previous work ${ }^{13}$ suggests that the Wigner approach can give a quite accurate description of the branching ratio for the reaction in Eq. (1). Thus, in a comparison to exact quantal results, it has already been demonstrated that the general trend of the energy-resolved (corresponding to a fixed wavelength of light) branching ratio is well reproduced by the Wigner method. This comparison did not, however, allow for a direct evaluation of the accuracy of the Wigner method since, in addition, a "classical" expression for the photodissociation cross sections was invoked. ${ }^{13}$

\section{THEORY}

A vertical electronic transition can be induced by a laser $\delta$ pulse: a replica of the initial nuclear state-times the transition dipole moment of the electronic transition-is created in the excited electronic state. ${ }^{16,17}$ Subsequent nuclear dynamics in the excited state is described by the FranckCondon wave packet

$$
|\phi(t)\rangle=\exp (-i \hat{H} t / \hbar)|\phi\rangle .
$$

Here $|\phi\rangle=\mu|\chi\rangle$, where $|\chi\rangle$ is the initial stationary nuclear state in the electronic ground state, $\mu$ is the projection of the electronic transition dipole moment on the polarization vector of the electric field of the laser, and $\hat{H}$ is the nuclear Hamiltonian of the excited electronic state.

We consider the two degree of freedom model of $\mathrm{HOD}^{13}$ where bending and overall rotation is neglected. The coordinates associated with the $\mathrm{O}-\mathrm{H}$ and $\mathrm{O}-\mathrm{D}$ bond lengths (local modes) are denoted by $r_{\mathrm{OH}}$ and $r_{\mathrm{OD}}$, respectively. The associated conjugate momenta are $p_{\mathrm{OH}}$ and $p_{\mathrm{OD}}$. The Hamiltonian is then given by

$$
H=\frac{p_{\mathrm{OH}}^{2}}{2 \mu_{\mathrm{OH}}}+\frac{p_{\mathrm{OD}}^{2}}{2 \mu_{\mathrm{OD}}}+\frac{p_{\mathrm{OH}} p_{\mathrm{OD}}}{m_{\mathrm{O}}} \cos \theta+V_{\theta}\left(r_{\mathrm{OH}}, r_{\mathrm{OD}}\right),
$$

where the reduced masses are $\mu_{\mathrm{OH}}=m_{\mathrm{H}} m_{\mathrm{O}} /\left(m_{\mathrm{H}}+m_{\mathrm{O}}\right)$ $=0.9481 \mathrm{amu}$ and $\mu_{\mathrm{OD}}=m_{\mathrm{D}} m_{\mathrm{O}} /\left(m_{\mathrm{D}}+m_{\mathrm{O}}\right)=1.7888 \mathrm{amu}$, and $\theta=104.52^{\circ}$ is the fixed bending angle.

The product of the wave function for the vibrational ground state and the transition dipole function can-to a good approximation-be written as a product of Gaussians in $\mathrm{O}-\mathrm{H}$ and $\mathrm{O}-\mathrm{D}$. This implies that the Wigner function takes the form ${ }^{13}$

$$
\begin{aligned}
\Gamma_{\phi}\left(r_{\mathrm{OH}}, r_{\mathrm{OD}}, p_{\mathrm{OH}}, p_{\mathrm{OD}}\right)=\frac{1}{(\pi \hbar)^{2}} \exp \left[-2 \alpha_{\mathrm{H}}\left(r_{\mathrm{OH}}-r_{\mathrm{OH}}^{\prime}\right)^{2}\right. \\
\left.-2 \alpha_{\mathrm{D}}\left(r_{\mathrm{OD}}-r_{\mathrm{OD}}^{\prime}\right)^{2}\right] \times \exp \left[-p_{\mathrm{OH}}^{2} /\left(2 \hbar^{2} \alpha_{\mathrm{H}}\right)\right. \\
\left.-p_{\mathrm{OD}}^{2} /\left(2 \hbar^{2} \alpha_{\mathrm{D}}\right)\right],
\end{aligned}
$$

where $r_{\mathrm{OH}}^{\prime}=0.97 \AA, r_{\mathrm{OD}}^{\prime}=0.96 \AA, \alpha_{\mathrm{H}}=67.28 \AA^{-2}$, and $\alpha_{\mathrm{D}}$ $=82.79 \AA^{-2}$. The Wigner functions for the two bonds are quite similar, the uncertainty in position/momentum for $\mathrm{O}-\mathrm{H}$ is slightly larger/smaller than for O-D. Thus, the standard deviation of the positions are $\Delta r_{\mathrm{OH}}=0.061 \AA$ and $\Delta r_{\mathrm{OD}}$ $=0.055 \AA$. Note that the average bond distance for $\mathrm{O}-\mathrm{H}$ is $0.97 \AA$ whereas it is $0.96 \AA$ for O-D.

In the Wigner method ${ }^{19}$ the time-evolution of each phase space point is given by Hamilton's equations. The potentialenergy surface $V_{\theta}\left(r_{\mathrm{OH}}, r_{\mathrm{OD}}\right)$ has been calculated by $a b$ initio methods, and an analytical fit has been constructed. ${ }^{22} \mathrm{We}$ have derived analytical expressions for the derivatives of the potential. The initial conditions are sampled according to the Wigner function. We use Gaussian importance sampling. All the results in the following are based on the sampling and time evolution of 10000 trajectories. The branching ratio is determined by the ratio between the number of trajectories that end up in each channel. The channels are separated by the line $r_{\mathrm{OD}}=r_{\mathrm{OH}}$. Figure 1(a) shows the potential and the initial probability density in the two bond coordinates corresponding to Eq. (4).

\section{RESULTS AND DISCUSSION}

The branching ratio obtained by exact quantum dynamics has been reported previously: $P_{\mathrm{H}}=0.76$ and $P_{\mathrm{D}}=0.24,{ }^{9}$ i.e., a branching ratio $P_{\mathrm{H}} / P_{\mathrm{D}}=3.17$. The result obtained using the Wigner method is $P_{\mathrm{H}}=0.73( \pm 0.01)$ and $P_{\mathrm{D}}$ $=0.27( \pm 0.01$ ) (where the numbers in parenthesis are a rough estimate of the statistical uncertainty). Thus, this result is in excellent agreement with exact quantum dynamics. Based on the motion of a swarm of trajectories, we can now address the question of how to understand/interpret the branching ratio.

In order to understand how the branching ratio depends on the initial state, we note that at time zero the FranckCondon wave packet sits slightly off the $45^{\circ}$ line in the H-OD channel [see Fig. 1(a)]. Is this asymmetry playing a role in determining the branching ratio? We have shifted the position of the initial state such that $r_{\mathrm{OH}}^{\prime}=r_{\mathrm{OD}}^{\prime}=0.97 \AA$. We find $P_{\mathrm{H}}=0.73$ and $P_{\mathrm{D}}=0.27$, that is, the branching ratio is unchanged. Similarly, due to the larger uncertainty in the $\mathrm{O}-\mathrm{H}$ distance compared to the $\mathrm{O}-\mathrm{D}$ distance, it is tempting to conclude that more trajectories start within the $\mathrm{H}+\mathrm{OD}$ channel, and that this proposition is an important factor in explaining that this is the preferred channel. ${ }^{11,13}$ However, the number of trajectories that start in each of the two channels is essentially the same, e.g., 55\% of the trajectories are starting in the $\mathrm{H}+\mathrm{OD}$ channel (when the position of the initial state is shifted such that $r_{\mathrm{OH}}^{\prime}=r_{\mathrm{OD}}^{\prime}$, exactly the same number of trajectories start in each channel).

Secondly, we evaluate the branching ratio when the initial momentum of all trajectories is set to zero. Previous attempts to rationalize that $\mathrm{H}+\mathrm{OD}$ is the preferred channel were based on qualitative arguments where the initial momentum is neglected. ${ }^{5,13}$ In Ref. 5 (Fig. 14) the regions of the potential-energy surface which lead to either $\mathrm{H}+\mathrm{OD}$ or $\mathrm{D}+\mathrm{OH}$ were mapped out. This mapping was based on classical dynamics for trajectories with no initial momentum. 

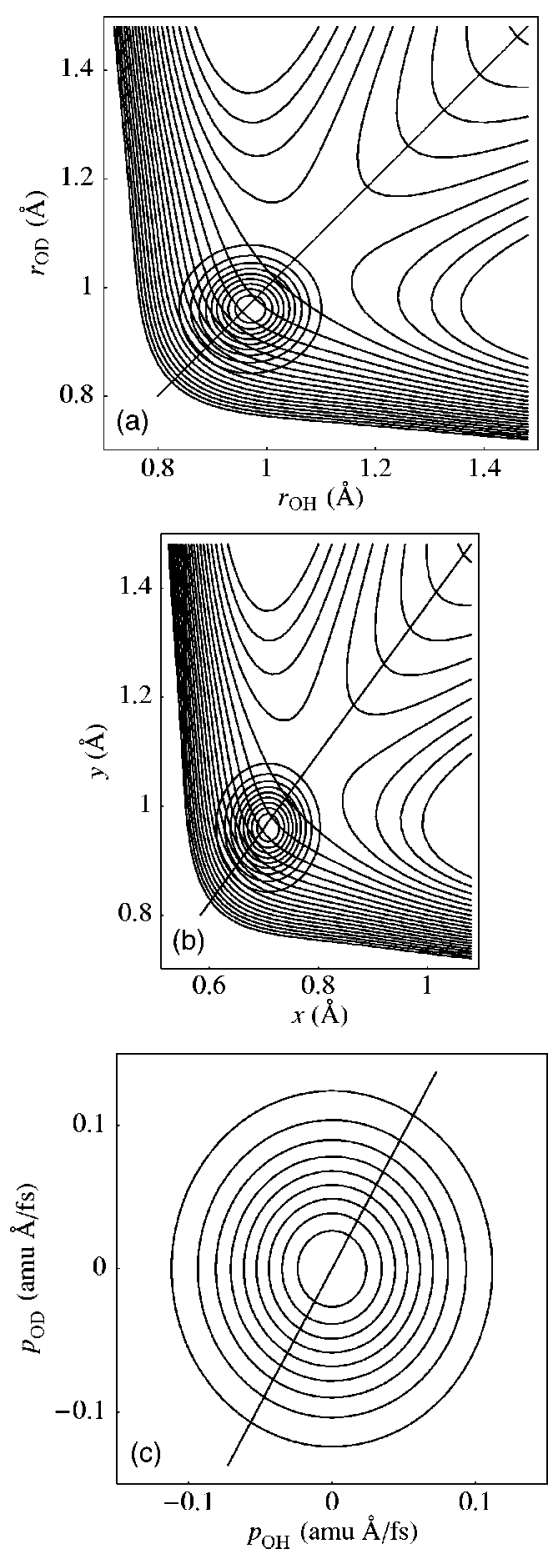

FIG. 1. Panel (a) shows the potential-energy surface associated with the first electronically excited state of water. The probability density of the initial state is superimposed. The straight line, $r_{\mathrm{OD}}=r_{\mathrm{OH}}$, separates the two reaction channels. Panel (b): same as (a), except that mass-weighted coordinates are used. Panel (c) shows the probability density of the initial momenta. The straight line, $p_{\mathrm{OD}}=\left(\mu_{\mathrm{OD}} / \mu_{\mathrm{OH}}\right) p_{\mathrm{OH}}$, is the dividing line for initial motion towards each of the two channels.

When the initial state is superimposed on the mapping, it is clear that $\mathrm{H}+\mathrm{OD}$ is the preferred channel. However, this seems not to give a branching ratio of 3 , but a much higher number. Indeed, we find $P_{\mathrm{H}}=0.96$ and $P_{\mathrm{D}}=0.04$. That is, the branching ratio is 24 . Thus, when the initial momentum distribution is neglected, the branching ratio is severely overestimated.

When interpreting the trajectory dynamics we note that motions along the $\mathrm{O}-\mathrm{H}$ and $\mathrm{O}-\mathrm{D}$ bonds are subject to the same forces, but that the motion along $\mathrm{O}-\mathrm{H}$ is faster (the acceleration is larger) due to the smaller reduced mass. Introduction of mass-weighted coordinates simplifies the discussion of dynamics in the sense that one can "simulate the actual trajectory by the process of rolling a ball on the

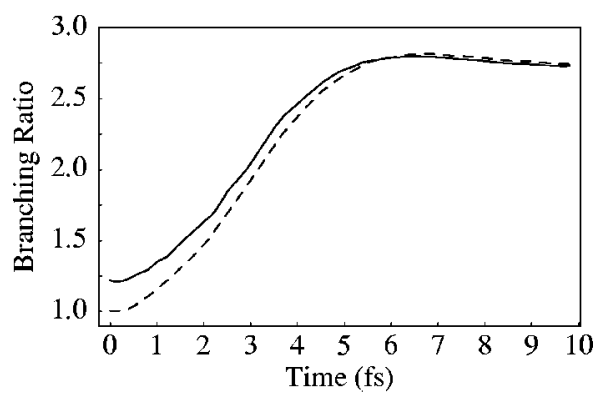

FIG. 2. The number of trajectories in the H+OD channel divided by the number of trajectories in the $\mathrm{D}+\mathrm{OH}$ channel as a function of time. The solid line corresponds to the exact sampling using Eq. (4), whereas the dashed line corresponds to sampling according to Eq. (5).

potential-energy surface." ${ }^{23}$ This is true because a common mass is assigned to the motion in the two degrees of freedom and the momentum coupling is eliminated by skewing the two bond distances to one another. However, in the HOD system the momentum coupling is very weak [leaving out the third term in Eq. (3) has negligible effect on numerical results] and we can therefore consider the kinetic energy to be already diagonal. Thus, we introduce the coordinates $x$ $=\left(\mu_{\mathrm{OH}} / \mu_{\mathrm{OD}}\right)^{1 / 2} r_{\mathrm{OH}}$ and $y=r_{\mathrm{OD}}$ with the conjugate momenta $p_{x}=\left(\mu_{\mathrm{OD}} / \mu_{\mathrm{OH}}\right)^{1 / 2} p_{\mathrm{OH}}$ and $p_{y}=p_{\mathrm{OD}}$. The kinetic energy then takes the (approximate) form $\left(2 \mu_{\mathrm{OD}}\right)^{-1}\left(p_{x}^{2}+p_{y}^{2}\right)=\left(\mu_{\mathrm{OD}} / 2\right)$ $\times\left(\dot{x}^{2}+\dot{y}^{2}\right)$. The dividing line between the two channels then becomes $y=\left(\mu_{\mathrm{OD}} / \mu_{\mathrm{OH}}\right)^{1 / 2} x$. The potential-energy surface in the mass-weighted coordinates is shown in Fig. 1(b). Note the asymmetry of the potential-energy surface [with a much steeper inner wall in the $x$ direction $(\mathrm{O}-\mathrm{H}$ bond) than in the $y$ direction (O-D bond)] and that the effective force [gradient of the two-dimensional (2D) potential-energy surface] in almost the entire "interaction region"-in particular, on the dividing line-points towards the preferred $\mathrm{H}+\mathrm{OD}$ channel. This explains the very large branching ratio obtained when the initial momentum distribution is neglected.

Next, we evaluate the branching ratio when the initial uncertainty in position is set to zero. Furthermore, to simplify the discussion, we start all trajectories at $r_{\mathrm{OH}}^{\prime}=r_{\mathrm{OD}}^{\prime}$ $=0.97 \AA$. We find now $P_{\mathrm{H}}=0.74$ and $P_{\mathrm{D}}=0.26$. This result is in excellent agreement with the exact result and we therefore conclude that the key feature of the initial state is the momentum distribution while the initial uncertainty in position and the slight asymmetry in the center of the Franck-Condon packet play negligible roles. In other words, the correct branching ratio is essentially reproduced by running trajectories sampled from the distribution

$$
\begin{aligned}
\widetilde{\Gamma}_{\phi}\left(r_{\mathrm{OH}}, r_{\mathrm{OD}}, p_{\mathrm{OH}}, p_{\mathrm{OD}}\right) \sim & \delta\left(r_{\mathrm{OH}}-r^{\prime}\right) \delta\left(r_{\mathrm{OD}}-r^{\prime}\right) \\
& \times \exp \left[-p_{\mathrm{OH}}^{2} /\left(2 \hbar^{2} \alpha_{\mathrm{H}}\right)\right. \\
& \left.-p_{\mathrm{OD}}^{2} /\left(2 \hbar^{2} \alpha_{\mathrm{D}}\right)\right],
\end{aligned}
$$

where $r^{\prime}=0.97 \AA$.

The relevant dynamics is very fast. Figure 2 shows the ratio between the number of trajectories in each of the two channels as a function of time (the asymptotic value being the branching ratio) for the two sampling functions, Eqs. (4) and (5). The results are very similar-confirming the above 

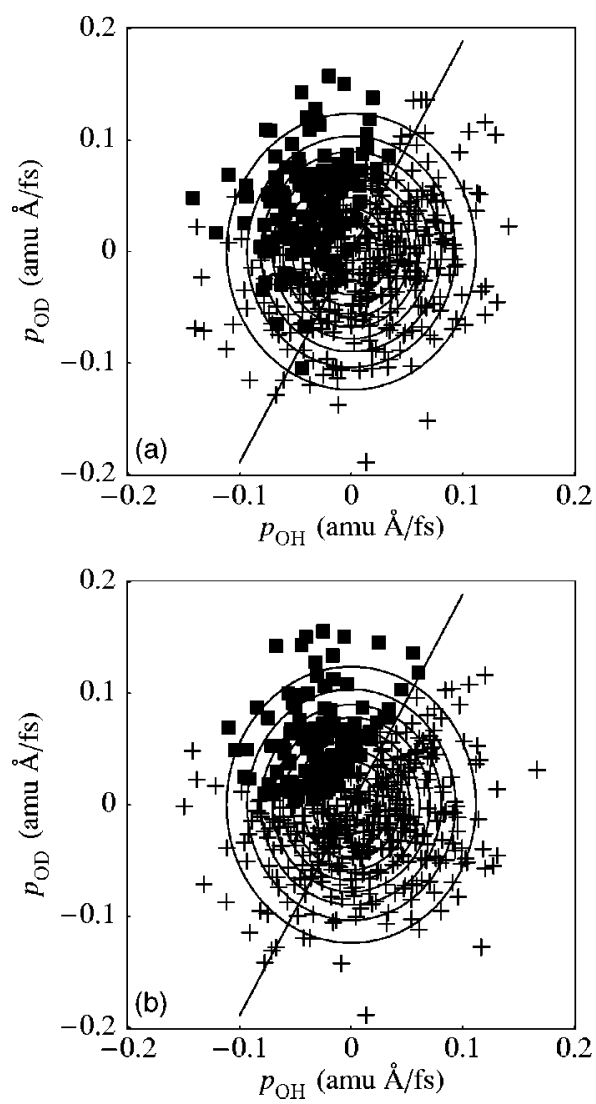

FIG. 3. The initial momenta (in the unit amu $\AA /$ fs) leading to the two channels, $\mathrm{H}+\mathrm{OD}(+)$ and $\mathrm{D}+\mathrm{OH}(\boldsymbol{\square})$. In panel (a) the exact sampling function in Eq. (4) is used, whereas sampling according to Eq. (5) is used in panel (b).

conclusion-with a small difference at early times due to the slight asymmetry in the center of the former distribution.

To obtain additional insight we analyze the values of the initial momenta which lead to $\mathrm{H}+\mathrm{OD}$ and $\mathrm{D}+\mathrm{OH}$, respectively. Note that the dividing line for initial motion towards each of the two channels is $\dot{r}_{\mathrm{OD}}=\dot{r}_{\mathrm{OH}}$, which becomes $p_{\mathrm{OD}}$ $=\left(\mu_{\mathrm{OD}} / \mu_{\mathrm{OH}}\right) p_{\mathrm{OH}}$. This is illustrated in Fig. 1(c). Figure 3 shows the initial momenta leading to the two channels, respectively, for the two sampling functions, Eqs. (4) and (5). Panels (a) and (b) show qualitatively the same, namely, (1) that trajectories that move initially toward the H+OD channel end up in that channel and (2) that of the trajectories that move initially toward the $\mathrm{D}+\mathrm{OH}$ channel, essentially, only trajectories with positive momentum along the O-D bond end up in that channel. Trajectories that move initially toward the $\mathrm{D}+\mathrm{OH}$ channel with negative momentum along the $\mathrm{O}-\mathrm{D}$ bond cross the dividing line to end up in $\mathrm{H}+\mathrm{OD}$ channel. In Fig. 4 we show the initial marginal densities in the two momentum coordinates $p_{\mathrm{OD}}$ and $p_{\mathrm{OH}}$ decomposed on the two channels. These are obtained from the data in Fig. 3(a) by integration for each channel over $p_{\mathrm{OD}}$ and $p_{\mathrm{OH}}$, respectively. We observe that the overlap between the momentum distributions for trajectories leading to $\mathrm{H}+\mathrm{OD}$ is larger than for the $\mathrm{D}+\mathrm{OH}$ channel, and the formation of $\mathrm{D}+\mathrm{OH}$ is, predominantly, associated with a positive value of $p_{\mathrm{OD}}$.

A rough estimate of the branching ratio can now be calculated from
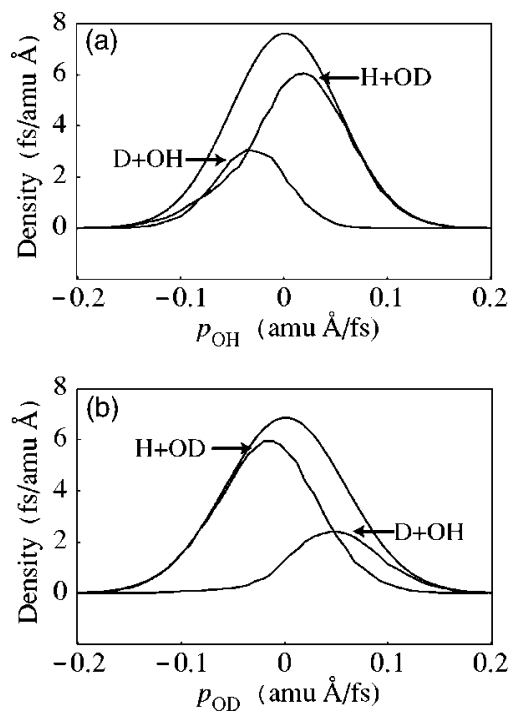

FIG. 4. Marginal probability densities for the initial momenta associated with the two channels $\mathrm{H}+\mathrm{OD}$ and $\mathrm{D}+\mathrm{OH}$ obtained from the data in Fig. $3(\mathrm{a})$.

$$
P_{\mathrm{D}} \sim \int_{\Omega} d r_{\mathrm{OH}} d r_{\mathrm{OD}} d p_{\mathrm{OH}} d p_{\mathrm{OD}} \Gamma_{\phi}
$$

where $\Omega$ is the part of phase space defined by $p_{\mathrm{OD}}$ $>\left(\mu_{\mathrm{OD}} / \mu_{\mathrm{OH}}\right) p_{\mathrm{OH}}$ and $p_{\mathrm{OD}}>0$. This gives a branching ratio of approximately 2 , which is just slightly underestimating the actual branching ratio.

Figure 5 shows four typical trajectories in massweighted coordinates. The trajectory that is reflected on the inner wall of the potential-energy surface in the $x$ direction ( $\mathrm{OH}$ bond) end up in the $\mathrm{H}+\mathrm{OD}$ channel because it is subject upon reflection to a force pointing in that direction.

If the trajectories only felt the repulsive forces pointing directly into one of the channels, we would expect a branching ratio close to 1 . We can test this proposition by shifting the initial state to the saddle point, i.e., $r_{\mathrm{OH}}^{\prime}=r_{\mathrm{OD}}^{\prime}=1.09 \AA$.

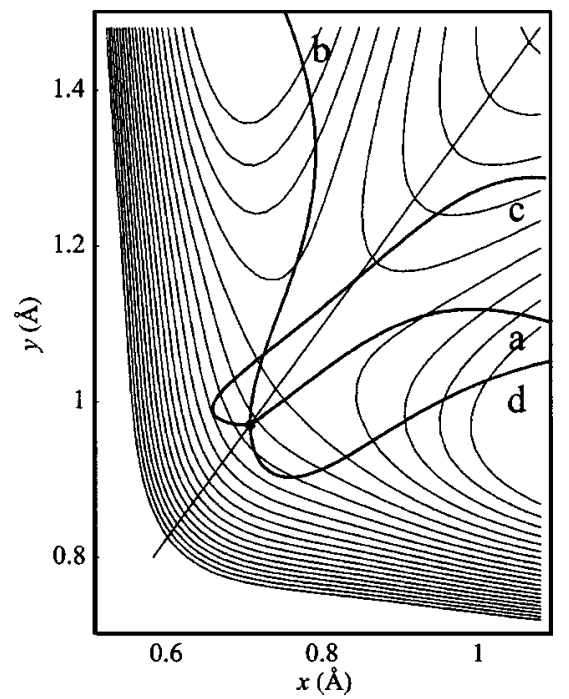

FIG. 5. Four typical trajectories in mass-weighted coordinates. All trajectories start at the same point $r_{\mathrm{OH}}^{\prime}=r_{\mathrm{OD}}^{\prime}=0.97 \AA$, but with different initial momenta. The initial momenta $\left(p_{x}, p_{y}\right)$ (in the unit amu $\AA /$ fs) are (a) 0,0 (b) $0,0.1$ (c) $-0.1,0$, and (d) $0,-0.1$. 

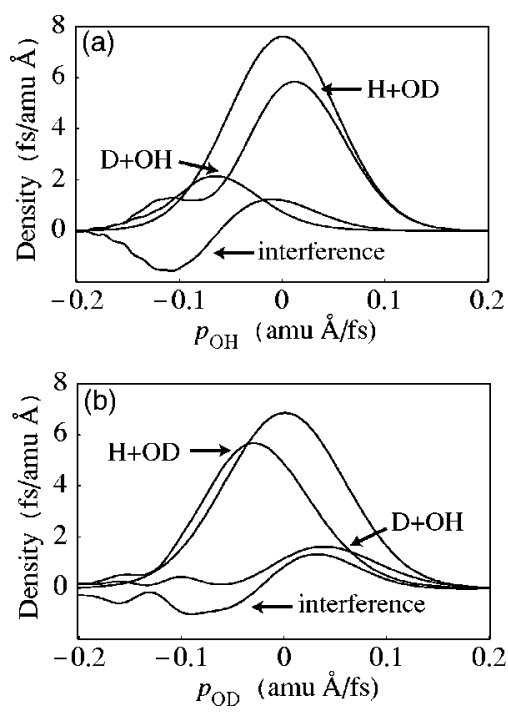

FIG. 6. Marginal probability densities for the initial momenta associated with the two channels $\mathrm{H}+\mathrm{OD}$ and $\mathrm{D}+\mathrm{OH}$ based on exact quantum dynamics, defined in Eq. (7), as well as the interference term defined in Eq. (8).

We get $P_{\mathrm{H}}=0.58$ and $P_{\mathrm{D}}=0.42$. Indeed, the branching ratio is now 1.38 and less than half of the value for the FranckCondon wave packet.

Hence, in a study with a small statistical uncertainty on the sampling of trajectories, we have shown that the Wigner method gives an excellent description of the branching ratio of $\mathrm{OH} / \mathrm{OD}$ bond breaking in HOD. The branching ratio is determined by the ultrafast dynamics which takes place within a few femtoseconds. In other words, the slight asymmetry in the initial state plays no role, which implies that the symmetry breaking (branching ratio different from $\sim 1$ ) is due to the dynamics. Indeed, we found that only trajectories with an initial momentum pointing towards the $\mathrm{D}+\mathrm{OH}$ channel and with a positive momentum along the O-D bond, actually end up in that channel. Ignoring the initial momentum distribution severely overestimates the branching ratio while neglecting the initial distribution in bond lengths has practically no effect. Pictorially speaking, the above findings can be summarized as follows: The formation of $\mathrm{D}+\mathrm{OH}$ arises almost exclusively from initial conditions where OD stretches faster than $\mathrm{OH}$, including initial conditions where $\mathrm{OD}$ stretches and $\mathrm{OH}$ contracts. All other situations lead to the formation of $\mathrm{H}+\mathrm{OD}$. Clearly, the main conclusions can be extended beyond HOD, to the isotope effect related to the fragmentation of symmetric molecules on a repulsive surface.

The above analysis was based on the classical motion of a swarm of trajectories sampled according to a Wigner function, i.e., a quasiprobability density in phase space. Finally, we ask whether we get a similar picture from exact quantum dynamics when we analyze state vectors in position and momentum space. We propagate the initial state $|\phi\rangle$, until the two "channel states" can be identified, i.e., $|\phi(t)\rangle$ $\rightarrow\left|\phi_{\mathrm{OD}}(t)\right\rangle+\left|\phi_{\mathrm{OH}}(t)\right\rangle$, corresponding to the formation of $\mathrm{H}$ $+\mathrm{OD}$ and $\mathrm{D}+\mathrm{OH}$, respectively. Then, we propagate each part in this decomposition backwards in time to $t=0$ and analyze the probability densities associated with each of these states neglecting, for the moment, possible interference terms. Note that the two parts in the decomposition are by construction orthogonal at all times, i.e., $\left\langle\phi_{\mathrm{OD}}(t) \mid \phi_{\mathrm{OH}}(t)\right\rangle$ $=0$, but interference terms may show up in the total probability densities [cf. Eq. (8)]. We observe the following: The densities in position space, i.e., $\left|\left\langle r_{\mathrm{OH}}, r_{\mathrm{OD}} \mid \phi_{\mathrm{OD}}(0)\right\rangle\right|^{2}$ and $\left|\left\langle r_{\mathrm{OH}}, r_{\mathrm{OD}} \mid \phi_{\mathrm{OH}}(0)\right\rangle\right|^{2}$ are very similar, whereas the corresponding densities in momentum space are qualitatively different. These findings confirm that it is the initial distribution of momenta which plays a decisive role for the branching ratio. In order to make the analysis more quantitative we show in Fig. 6 the marginal probability densities in the momenta for the two initial channel states,

$$
\begin{aligned}
& f_{i}\left(p_{\mathrm{OH}}\right)=\int_{-\infty}^{\infty}\left|\left\langle p_{\mathrm{OH}}, p_{\mathrm{OD}} \mid \phi_{i}(0)\right\rangle\right|^{2} d p_{\mathrm{OD}}, \\
& f_{i}\left(p_{\mathrm{OD}}\right)=\int_{-\infty}^{\infty}\left|\left\langle p_{\mathrm{OH}}, p_{\mathrm{OD}} \mid \phi_{i}(0)\right\rangle\right|^{2} d p_{\mathrm{OH}},
\end{aligned}
$$

where $i=\mathrm{OH}$ or OD. Comparing panels (a) and (b), we observe that in the initial state leading to $\mathrm{D}+\mathrm{OH}$, the distribution of momenta associated with the two bonds is quite different and dominated by positive momenta along the OD bond, in agreement with the previous results (cf. Fig. 4).

The results associated with state vectors should, however, be interpreted with some caution due to the presence of interference terms between the two parts in the decomposition of the initial state. Thus, since $|\phi\rangle=\left|\phi_{\mathrm{OD}}(0)\right\rangle+\left|\phi_{\mathrm{OH}}(0)\right\rangle$, the probability density associated with the initial state is

$$
\begin{aligned}
\left|\left\langle p_{\mathrm{OH}}, p_{\mathrm{OD}} \mid \phi\right\rangle\right|^{2}= & \left|\left\langle p_{\mathrm{OH}}, p_{\mathrm{OD}} \mid \phi_{\mathrm{OD}}(0)\right\rangle\right|^{2} \\
& +\left|\left\langle p_{\mathrm{OH}}, p_{\mathrm{OD}} \mid \phi_{\mathrm{OH}}(0)\right\rangle\right|^{2}+\text { interference. }
\end{aligned}
$$

Figure 6 shows that the interference terms are nonnegligible and they contain some oscillating positive and negative contributions, reminiscent of the undulations in the quantum densities. Note that the three terms in Eq. (8), of course, add up to the Gaussian momentum distributions [see Eq. (4)] associated with the initial state.

\section{ACKNOWLEDGMENT}

This work was supported by the Danish Natural Science Research Council.

${ }^{1}$ S. A. Rice and M. Zhao, Optical Control of Molecular Dynamics (Wiley, New York, 2000).

${ }^{2}$ P. Brumer and M. Shapiro, Annu. Rev. Phys. Chem. 43, 257 (1992).

${ }^{3}$ N. E. Henriksen, Chem. Soc. Rev. 31, 37 (2002).

${ }^{4}$ V. Engel, V. Staemmler, R. L. Vander Wal et al., J. Phys. Chem. 96, 3201 (1992).

${ }^{5}$ J. Zhang, D. G. Imre, and J. H. Frederick, J. Phys. Chem. 93, 1840 (1989).

${ }^{6}$ I. Bar, Y. Cohen, D. David, S. Rosenwaks, and J. J. Valentini, J. Chem. Phys. 93, 2146 (1990).

${ }^{7}$ R. L. Vander Wal, J. L. Scott, and F. F. Crim, J. Chem. Phys. 92, 803 (1990).

${ }^{8}$ R. L. Vander Wal, J. L. Scott, F. F. Crim, K. Weide, and R. Schinke, J. Chem. Phys. 94, 3548 (1991).

${ }^{9}$ B. Amstrup and N. E. Henriksen, J. Chem. Phys. 97, 8285 (1992).

${ }^{10}$ N. E. Henriksen and B. Amstrup, Chem. Phys. Lett. 213, 65 (1993). 
${ }^{11}$ K. Mishima and K. Yamashita, J. Chem. Phys. 110, 7756 (1999).

${ }^{12}$ N. Elghobashi, P. Krause, J. Manz, and M. Oppel, Phys. Chem. Chem. Phys. 5, 4806 (2003).

${ }^{13}$ V. Engel and R. Schinke, J. Chem. Phys. 88, 6831 (1988).

${ }^{14}$ N. Shafer, S. Satyapal, and R. Bersohn, J. Chem. Phys. 90, 6807 (1989).

${ }^{15}$ S. Goursaud, M. Sizun, and F. Fiquet-Fayard, J. Chem. Phys. 65, 5453 (1976).

${ }^{16}$ E. J. Heller, Acc. Chem. Res. 14, 368 (1981).

${ }^{17}$ N. E. Henriksen, Adv. Chem. Phys. 91, 433 (1995).
${ }^{18}$ P. Brumer and M. Shapiro, Chem. Phys. 139, 221 (1989).

${ }^{19}$ E. J. Heller, J. Chem. Phys. 65, 1289 (1976).

${ }^{20}$ N. E. Henriksen, V. Engel, and R. Schinke, J. Chem. Phys. 86, 6862 (1987).

${ }^{21}$ K. B. Møller, J. P. Dahl, and N. E. Henriksen, J. Phys. Chem. 98, 3272 (1994).

${ }^{22}$ V. Engel, R. Schinke, and V. Staemmler, J. Chem. Phys. 88, 129 (1988).

${ }^{23}$ R. D. Levine and R. B. Bernstein, Molecular Reaction Dynamics and Chemical Reactivity (Oxford, New York, 1987). 